\title{
THE DIAMETER AND THE MEAN DISTANCE OF A RIEMANNIAN MANIFOLD
}

\author{
BOJAN MOHAR
}

Let $M$ be a compact Riemannian manifold. For $x, y \in M$, denote by $\rho(x, y)$ the distance between $x$ and $y$, i.e., the infimum of lengths of continuous piecewise $C^{1}$ paths between $x$ and $y$. (Notice that for geodesically complete manifolds there is always a path from $x$ to $y$ of length $\rho(x, y)$.) The invariants of $M$ based on the distance $\rho$ are called metrical invariants. Most of them are related to the geometry of $M$. Let us mention a few of them.

The diameter, $\operatorname{diam}(M)$, of $M$ is the maximal value of $\rho(x, y)$ for $x, y \in$ $M$. If two points $x, y$ are at distance $\operatorname{diam}(M)$, they are called antipodal. Denote by

$$
\bar{\rho}(x, M)=\frac{1}{\operatorname{Vol}(M)} \int_{M} \rho(x, y) d V(y)
$$

the average distance of points in $M$ from $x$. Then

$$
\bar{\rho}(M)=\frac{1}{\operatorname{Vol}(M)} \int_{M} \bar{\rho}(x, M) d V(x)
$$

is called the mean distance of $M$. These distances are important metric invariants of $M$, but, surprisingly enough, they have not been extensively investigated yet.

Denote by $\Delta$ the Laplace-Beltrami differential operator (Laplacian for short) on $M$. It is well known that the spectrum of $\Delta$ is a discrete set of real numbers, $\lambda_{0}=0<\lambda_{1} \leq \lambda_{2} \leq \lambda_{3} \leq \ldots$. The first nontrivial eigenvalue $\lambda_{1}=\lambda_{1}(M)$ of the Laplacian is related to many geometric properties of $M$. In this note we present inequalities between the diameter, or the mean distance of $M$, and $\lambda_{1}(M)$. For $\delta>0$, let $B(x, \delta)$ denote the ball of radius $\delta$ centered at $x \in M$, and let

$$
v(M, \delta):=\min _{x \in M} \operatorname{Vol}(B(x, \delta)) .
$$

THEOREM 1. Let $M$ be a compact Riemannian manifold with the first nontrivial eigenvalue of the Laplace operator on $M$ equal to $\lambda_{1}$. Then for any $\delta>0$,

$$
\operatorname{diam}(M) \leq 2\left(\delta+\frac{2}{\sqrt{\lambda_{1}}}\left\lceil\log _{2} \frac{\operatorname{Vol}(M)}{2 v(M, \delta)}\right\rceil\right) .
$$

Received by the editors March 8, 1989.

1980 Mathematics Subject Classification (1985 Revision). Primary 53C20, 53C30.

This work was supported in part by the Research Council of Slovenia, Yugoslavia.

(C) 1989 American Mathematical Society $0273-0979 / 89 \$ 1.00+\$ .25$ per page 
THEOREM 2. Under the same assumptions as above and for arbitrary $x \in M$,

$$
\bar{\rho}(x, M)<\delta+\frac{2}{\sqrt{\lambda_{1}}}\left\lceil\log _{2} \frac{\operatorname{Vol}(M)}{v(M, \delta)}\right]+\frac{\delta v(M, \delta)}{\operatorname{Vol}(M)} .
$$

Of course, (4) gives rise to the same upper bound on the mean distance $\bar{\rho}(M)$.

The main idea in the proofs of both theorems is the same. We sketch only the slightly simpler proof of Theorem 1 . The details will appear elsewhere.

The main step in proving Theorem 1 is to show that large $\lambda_{1}$ forces fast growth of the manifold: By the Rayleigh's Theorem for the Laplacian $\Delta$ on $M$,

$$
\lambda_{1}=\min \left\{\frac{(\Delta f, f)}{(f, f)} \mid f \in C^{2}(M), \int_{M} f=0, f \neq 0\right\}
$$

where $(f, g)=\int_{M} f g$. This implies a similar, handier formula

$$
\lambda_{1}=2 \operatorname{Vol}(M) \min _{g \in \Phi} \frac{(\Delta g, g)}{\int_{M} \int_{M}[g(x)-g(y)]^{2} d V(x) d V(y)}
$$

where $\Phi$ is the set of all nonconstant $C^{2}$ functions on $M$.

Let $N$ be a compact submanifold of $M$ of codimension one which divides $M$ into two disjoint open submanifolds $M_{1}, M_{2}$ with common boundary $N=\partial M_{1}=\partial M_{2}$. For $\varepsilon>0$, let $N_{\varepsilon}:=\left\{x \in M_{2} \cup N \mid \rho(x, N) \leq \varepsilon\right\}$. Applying the function

$$
g(x)= \begin{cases}0, & x \in M_{1}, \\ \frac{\rho(x, N)}{\varepsilon}, & x \in N_{\varepsilon}, \\ 1, & x \in M_{2} \backslash N_{\varepsilon},\end{cases}
$$

in (5) one obtains, after some computation, that if $\operatorname{Vol}\left(M_{1}\right) \leq \operatorname{Vol}\left(M_{2} \backslash N_{\varepsilon}\right)$,

$$
\varepsilon^{2} \lambda_{1}<\frac{\operatorname{Vol}(M) \operatorname{Vol}\left(N_{\varepsilon}\right)}{\operatorname{Vol}\left(M_{1}\right)\left[\operatorname{Vol}\left(M_{2}\right)-\frac{1}{2} \operatorname{Vol}\left(N_{\varepsilon}\right)\right]} .
$$

If, moreover, $\operatorname{Vol}\left(M_{2} \backslash N_{\varepsilon}\right) \geq \frac{1}{2} \operatorname{Vol}(M)$ then (6) implies for $\varepsilon=\frac{2}{\sqrt{\lambda_{1}}}$ that

$$
\operatorname{Vol}\left(M_{1} \cup N_{\varepsilon}\right)>2 \operatorname{Vol}\left(M_{1}\right) \text {. }
$$

It is a matter of routine to derive (3) from (7). It should be mentioned that (7) also implies (4) but some additional work is required.

The obtained inequalities, upper bounds on $\operatorname{diam}(M)$ and $\bar{\rho}(M)$, hold in general, whereas in previous works a similar result was obtained by Burger and Schroeder [BS] in the case only, when $M$ is a compact quotient of $\mathbf{H}_{F}^{n}$, F being one of the rings $\mathbf{R}, \mathbf{C}, \mathbf{H}$, or $\mathbf{O}$ (this one for $n=2$ only). Another work [B] uses the results of [BS] and some further analysis to obtain bounds on the diameter of a compact Riemannian manifold and its covers. 


\section{REFERENCES}

[B] R. Brooks, Some remarks on volume and diameter of Riemannian manifolds, J. Differential Geom. 27 (1988), 81-86.

[BS] M. Burger and V. Schroeder, Volume, diameter, and the first eigenvalue of locally symmetric spaces of rank one, J. Differential Geom. 26 (1987), 273-284.

DePartment OF MATHEMATICS, UNIVERSity OF LJUBLJANA, JADRANSKA 19, 61111 LJUBLJana, Yugoslavia 
